\title{
CD23,
}

\section{une molécule plurifonctionnelle}

Les connaissances sur le CD23, récepteur de faible affinité pour les IgE, ont progressé considérablement depuis quelques années. Cette molécule, sous forme de protéine membranaire ou de fragments solubles circulants, présente des activités biologiques multiples, spécifiques des types cellulaires qui l'expriment. Son implication est maintenant reconnue dans des processus variés comme la prolifération des lymphocytes $B$, la présentation de l'antigène, la défense antiparasitaire, la différenciation de certains précurseurs hématopoiétiques et la régulation de la synthèse des IgE. Ce dernier effet pourrait être utilisé dans le contrôle thérapeutique de l'allergie.

\section{Jean-Pierre Kolb Élisabeth Génot Vincent Lagente Bernard Dugas}

a caractérisation de la molécule CD23 (CD : cluster of differentiation) a été le fruit de recherches menées dans des disciplines différentes. Les travaux des groupes de Spiegelberg, Metzger, Ishizaka et Capron avaient établi l'existence, à la surface des cellules lymphoïdes et myélomonocytaires, d'un récepteur de faible affinité (FceRII) pour le fragment Fc des immunoglobulines $\mathrm{E}$ (IgE). Celui-ci était distinct du récepteur de haute affinité, complexe multicaténaire appelé $F c \in R I$, exprimé sur les mastocytes et les basophiles [1]. Par ailleurs, la molécule CD23, définie par sa réactivité vis-à-vis d'un ensemble d'anticorps monoclonaux, avait été identifiée comme un antigène d'activation exprimé à la surface des lymphocytes $B$ après infec- tion par le virus d'Epstein-Barr (EBV), d'où son premier nom d'EBVCS ( $E B V$ cell surface antigen) ou de BLAST-2. L'identité entre les deux molécules fut établie lorsqu'il fut montré que les anticorps monoclonaux définissant le CD23 réagissaient avec la molécule recombinante codée par l'ADNc du FceRII.

\section{Structure de la molécule}

Le gène du CD23 humain a été cloné par trois groupes indépendants [2-4] et ne présente pas de similitude avec ceux codant pour le $F c \in R I$ (chaîne $\alpha$ ) et le récepteur Fc des IgA, qui appartiennent à la superfamille des immunoglobulines. La molécule de 321 acides aminés, sans séquence signal, est une protéine membranaire 
de type II*, avec un seul domaine transmembranaire et un domaine extracellulaire constitué de la partie C-terminale (figure 1). Cette portion contient un domaine présentant une forte analogie de séquence avec des lectines animales de type $\mathrm{C}^{* *}$. Le poids moléculaire déduit de la séquence est de 36000 , alors que la forme naturelle est de $45 \mathrm{kDa}$. Le CD23 humain présente un site unique de $\mathrm{N}$-glycosylation qui joue un rôle important en ralentissant le catabolisme de la molécule. Le gène, dont il n'existe qu'une seule copie, a été localisé sur le chromosome 19 et comporte 11 exons. Il existe une série de trois exons très homologues (exons répétés 5,6 et 7 ) codant chacun pour 21 acides aminés et dont le rôle biologique n'est pas connu. Les exons 9 à 11 codent pour le domaine d'homologie des lectines qui inclut quatre cystéines parfaitement conservées et qui contient le site de liaison à l'IgE. A la différence de ces lectines et bien que l'IgE soit une molécule riche en sucre, le CD23 ne reconnaît pas son ligand via une structure glycosylée, mais interagit au niveau d'un épitope thermolabile d'une dizaine d'acides aminés, situé dans le domaine $\mathrm{CH}_{3}$ de $\operatorname{l} \mathrm{IgE}^{* * *}$. Ce site est très proche de celui reconnu par le FceRI, ce qui explique que des concentrations élevées de la forme soluble du CD23 puissent déplacer l'IgE du récepteur $F c \in R I$. $\mathrm{Ce}$ dernier a pour son ligand une affinité forte $\left(\mathrm{Kd}=10^{-9} \mathrm{M}\right)$, alors que le CD23 présente une affinité beaucoup plus faible $(\mathrm{Kd}=$ $10^{-7} \mathrm{M}$ à $10^{-8} \mathrm{M}$, suivant le degré d'oligomérisation de l' $\mathrm{IgE}$ ), toutefois

* Les protéines membranaires de type I et 11 ont un seul domaine transmembranaire et leur extrémité $N$ terminale est extracellulaire (type I) ou intracytoplasmique (type II). Quant aux protéines de type III, elles ont des domaines transmembranaires multiples. ** Protéines (non enzymatiques, non immunes) reconnaissant des glucides et dont la liaison requiert la présence de calcium (le prototype en est le récepteur de l'asialo-glycoprotéine.

*** Le domaine CH3 de l'IgE est le troisième domaine de la portion constante de la chaine lourde ( $\epsilon)$ de l'IgE.

$m / s n^{\circ} 5$, vol. 7, mai 91

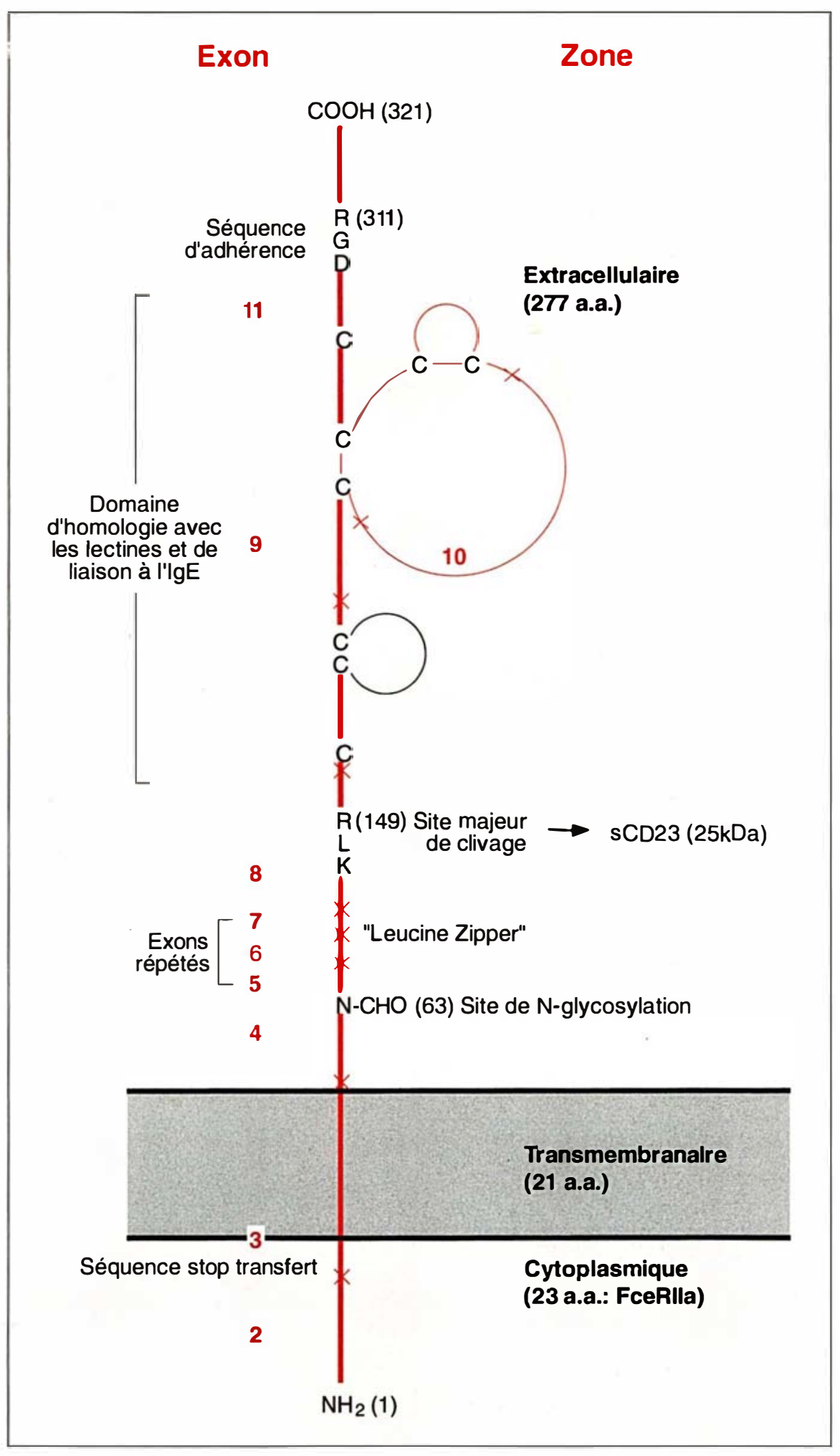

Figure 1. Structure de la molécule CD23 (Fce RIla) humaine. Les $X$ représentent les limites correspondant aux différents exons; l'exon 1 n'est pas traduit. La " séquence stop transfert " est le signal qu'indique la position de l'arrêt de la translocation de la protéine à travers la membrane cellulaire. 


\section{RÉFÉRENCES}

1. Metzger H. Molecular aspects of receptors and binding factors for IgE. Adv Immunol $1988 ; 43: 277-312$

2. Kikutani H, Inui $\mathrm{S}$, Sato $\mathrm{R}$, et al. Molecular structure of human lymphocyte receptor for immunoglobulin E. Cell $1986 ; 47$ : 657-65.

3. Ikuta K, Takami M, Kim CW, et al. Human lymphocyte $\mathrm{Fc}$ receptor for $\mathrm{IgE}$ : sequence homology of its cloned cDNA with animal lectins. Proc Natl Acad Sci USA 1987 84: 819-23.

4. Lüdin C, Hof stetter H, Sarfati M, et al. Cloning and expression of the cDNA coding for a human lymphocyte $\mathrm{IgE}$ receptor. EMBO J 1987 ; 6 : 109-14.

5. Yokota A, Kikutani H, Tanaka $\mathrm{T}$, et al Two species of human Fce receptor II (FceRII/CD23): tissue-specific and IL-4-specific regulation of gene expression. Cell 1988; 55 : 611-8

6. Suter U, Bastos R, Hof stetter H. Molecular structure of the gene and the 5'flanking region of the human lymphocyte immunoglobulin E receptor. Nucleic Acids Res $1987 ; 15: 7295-308$

7. Gollnick SO, Trounstine ML, Yamashita LC, Kehry MR, Moore KW. Isolation, characterization and expression of cDNA clones encoding the mouse Fc receptor for IgE (Fce RII). I Immunol $1990 ; 144$ : 1974-82.

8. Delespesse G, Sarfati M, Hofstetter $H$. Human IgE-binding factors. Immunol Today $1989 ; 10: 159-64$

9. Letellier $\mathrm{M}$, Nakajima $\mathrm{T}$, PulidoCedujo G, Hofstetter H, Delespesse G. Mechanism of formation of human IgEbinding factors (soluble CD23). III. Evidence for a receptor (FceRII) associated proteolytic activity. J Exp Med $1990 ; 172$ : 693-700.

10. Bonnefoy JY, Guillot O, Spits H, Blanchard D, Ishizaka K, Banchereau J. The low-affinity receptor for IgE (CD23) on B lymphocytes is spatially associated with HLA-DR antigens. $J$ Exp Med $1988 ; 167$ : $57-72$

11. Nonaka M, Hsu DK, Hanson CM, Aosai F, Katz DH. Cloning of cDNA coding for low-affinity $\mathrm{Fc}$ receptors for $\mathrm{IgE}$ on human $\mathrm{T}$ lymphocytes. Int Immunol $1989 ; 1: 254-9$. comparable à celle de récepteurs Fc pour d'autres isotypes. Cela pose le problème de la reconnaissance éventuelle par la CD23 d'un autre ligand encore inconnu, différent de l'IgE, via un motif glucidique, et donc de sa possible bispécificité.

L'extrémité C-terminale contient, en configuration inversée, la séquence consensus RGD (Arg-Gly-Asp) d'adhérence de la fibronectine. Par ailleurs, il existe un motif putatif leucine zipper (région 95-116) dans une zone d'hélice $\alpha$ de la molécule, qui pourrait permettre la dimérisation du CD23 et/ou son association avec d'autres molécules membranaires.

Deux types de FceRII ont été identifiés qui diffèrent dans leur partie intracytoplasmique $\mathrm{N}$-terminale alors qu'ils partagent la même région $\mathrm{C}$ terminale extracellulaire. Ces deux espèces résultent d'un épissage alternatif du messager et de sites différents d'initiation de la transcription [5]. La forme FceRIIa est exprimée constitutivement dans les lymphocytes $B$ normaux et certaines lignées tumorales B. Dans les autres types cellulaires exprimant le CD23, soit spontanément, soit après activation, on ne détecte que la forme FceRIIb. Le promoteur du FceRIIa a été analysé ; il est encadré de deux paires de séquences Alu en répétition inversée et d'une paire d'un élément de $188 \mathrm{pb}$, aussi en répétition inversée. Deux segments régulateurs se trouvent en amont de ce promoteur, l'un à effet négatif, l'autre positif répondant à un facteur de transcription induit par l'IL-4 [6].

Le CD23 murin présente une similarité de structure et une homologie de séquence avec le CD23 humain [7]. Cependant la molécule murine, possédant quatre exons répétés, comporte 331 acides aminés ; elle a deux sites de $\mathrm{N}$-glycosylation et son extrémité C-terminale s'arrête juste avant la séquence RGD. Il n'existe qu'une isoforme, proche du type FceRIIa humain, ce qui expliquerait que, dans cette espèce, le CD23 n'ait été détecté presque exclusivement que dans les cellules $B$.
Tableau I

EXPRESSION ET FONCTIONS DU CD23 MEMBRANAIRE DANS DIVERS TYPES CELLULAIRES

Cellules Fonctions proposées

Lymphocytes B

Présentation de l'antigène aux cellules $T$ via les IgE spécifiques

Régulation de synthèse $d^{\prime} \lg E$

Récepteur de facteur de croissance ? sCD23 facteur de croissance autocrine?

Lymphocytes T

Contrôle de la prolifération

Monocytes/macrophages

Cytotoxicité de type ADCC

Défense antiparasitaire. Phagocytose

Libération de médiateurs de l'inflammation

Éosinophiles hypodenses

Cytotoxicité de type ADCC

Défense antiparasitaire

Plaquettes

Cytotoxicité de type ADCC

Défense antiparasitaire

Cellules de Langerhans

Présentation de l'antigène aux cellules $T$

Cellules folliculaires

dendritiques

Présentation de I'antigène aux cellules $T$ Sauvetage de I'apoptose des lymphocytes B via le sCD23

Cellules épithéliales thymiques Maturation des lymphocytes $T$, via le sCD23

Cellules stromales médullaires Maturation des lymphocytes $\mathrm{T}$ et de précurseurs myéloïdes via le sCD23 


\section{Fragments solubles du CD23}

Le CD23 donnc naissance, par unc séric de clivages séquentiels de sa forme membranaire, à une famille de fragments solubles. Ces fragments exerccnt des activités biologiques très variécs et sont dénommés sCD23 (soluble CD23) ou IgE-BF (IgE-binding factor) pour ccux d'entre eux qui conservent la capacité de lier l'IgE, avec une constante d'association d'environ $10^{6} \mathrm{M}^{-1}$ (le nom d'IgE-BF désignc également d'autres molécules sécrétées par des lymphocytes $T$, sans homologie structurelle avec le CD23 et qui cxercent aussi un rôle dans la régulation de la synthèse d'IgE [1, 8]). On détecte ainsi des fragments sCD23/IgE-BF de 37, 33 et $29 \mathrm{kDa}$ relativement instables, donnant finalement un fragment stable de $25 \mathrm{kDa}$. Il existe aussi un fragment de 12 à $15 \mathrm{kDa}$, incapable de lier l'IgE. Le processus serait autocatalytique, car la molécule entière et ses fragments solubles recombinants de 37 ct $29 \mathrm{kDa}$ clivent eux-mêmes le CD23 ; la fixation de l'IgE inhibe en partic cette activité enzymatique [9]. Des anticorps anti-CD23 ralentissent ce clivage, accéléré en revanche par des inhibiteurs de la $\mathrm{N}$-glycosylation. Un mécanisme complémentaire de clivage intraccllulairc à partir de la molécule entièrc a été décrit, mais sa contribution à la libération des facteurs solubles cst difficile à déterminer. Chez la souris, il existe aussi des formes solubles du CD23, mais leur liaison à $\mathrm{l}$ 'IgE cst très difficile à mettre en évidence.

\section{Expression membranaire du CD23 sur divers types cellulaires}

\section{Cellules normales}

- Lymphocytes B. Le CD23 est présent à faible densité sur la membrane des lymphocytes B normaux matures circulants, exclusivement sur des cellules $\operatorname{sgM} / \mathrm{sIgD}^{*}$ avant la phase de commutation isotypique. Il est spatialement associé, de façon non covalentc, aux antigènes HLA-DR du complexe majeur d'histocompatibilité $(\mathrm{CMH})$ à la surface des lymphocytes B humains [10]. Les cellules B médullaires sIgM/sIgD n'expriment le CD23 qu'après stimulation et cette augmentation du CD23 a permis de caractériser un état d'activation précoce appelé $\mathrm{Go}(\mathrm{a})$. Chcz l'homme, les lymphocytes B CD5 + et CD5expriment lc CD23. Ce marqueur CD5, présent sur les cellules de leucémie lymphoïde chronique B (LLCB), identifie des cellules B dont le répertoire est biaisé vers les antigènes du "soi ". Chez la souris, en revanche, les lymphocytes $\mathrm{B}$ Ly-1 + (équivalent murin de CD5) sont CD23 - , alors que la présence du CD23 paraît être un marqueur sélcctif des précurseurs des cellules B qui vont sécréter les $\mathrm{IgE}$.

Des cellules B isolées du sang de cordon expriment un niveau élevé de CD23. Cette sous-population, qui est décelée aussi chez des paticnts atteints d'immunodéficience combinée sévèrc, pourrait donc être présente durant l'ontogénie normale et disparaître lors de l'acquisition de la compétence immunitaire. Après greffe de moelle, autologue ou hétérologuc, on retrouve d'ailleurs des lymphocytes $B$ activés $\mathrm{CD} 23^{+}$, mais ceux-ci montrent un défaut fonctionnel à proliférer.

- Lymphocytes T. Le CD23 cst exprimé spontanément à la surface de lymphocytes $\mathrm{T}$ de patients ayant la maladie de Kimura** ou infectés par le virus du SIDA ct dans des clones $T$ transformés par le virus HTLV-1, où l'on détecte aussi l'ARNm. Par ailleurs, un ADNc du CD23 a été cloné à partir d'une lignéc T humaine HTLV-1 - ; cet ADNc, identique à cclui du CD23b, contient en plus une répétition de $64 \mathrm{pb}$ dans la région 3' non traduite [11]. Le CD23 est aussi exprimé, au moins transitoirement, sur une souspopulation de cellules $\mathrm{T}$ normales provenant d'amygdales [12].

- Monocytes/macrophages. Si les monocytes circulants et les macrophages alvéolaires humains normaux non stimulés n'expriment pas (ou très peu) le CD23, on détecte cette molé-

\footnotetext{
* La nomenclature généalement utilisée pour les immunoglobulines membranaires est $\mathrm{s} I \mathrm{~g}$, pour Ig de surface.

** Hyperimmunoglobulinémie $E$ non allergique.
}

cule sur les monocytes de patients préscntant un syndrome hypcr-IgE et sur les macrophages de paticnts asthmatiques ou attcints d'alvéolite extrinsèque. Chez les rongcurs, il existe un récepteur $\mathrm{F} c \in \mathrm{R}$ II à la surface des macrophages, mais son identification au CD23 n'cst pas encore claircment établie.

Éosinophiles. La présence d'un réceptcur IgE sur les éosinophiles hypodenses a été rapportéc par lc groupe de Capron ; il cxiste une antigénicité commune entre le CD23 des ccllules $\mathrm{B}$ et ce réccpteur, et des anticorps dirigés contre une séquence contenant le motif consensus d'adhérence RGD de type fibronectinc reconnaissent le FceRII sur les éosinophiles et les cellules $B$ [13]. L'identification du CD23 comme Fce RII des éosinophiles n'est toutcfois pas absolument établic et d'autres entités pourraient contribuer à la formation de ce récepteur, notamment la molécule Mac-2, unc lectine reconnaissant le galactose.

- Plaquettes. Il existe un FceRII sur les plaquettes humaines, dont l'expression est accrue en présence d'interféron $\gamma$. Cc réceptcur est impliqué dans la cytotoxicité antiparasitaire dépendantc d'IgE ct a été identifié commc étant le CD23 à l'aide d'anticorps monoclonaux.

- Cellules de Langerhans de la peau. Des récepteurs de type $\mathrm{Fc} \in \mathrm{RII}$, associés à l'antigène $\mathrm{CD} 1$, ont été détectés spontanément sur les cellules de Langerhans provenant de l'épiderme de patients attcints de dermatite atopique [14].

- Cellules folliculaires dendritiques. Le lignage de ces cellules cst encore mal défini, mais celles-ci, tout commc leur contrepartie tumorale, expriment à haute densité le CD23, qui jouerait un rôle important dans la présentation de l'antigène aux ccllules $T$ et la maturation terminale des lymphocytes B.

- Cellules épithéliales. La présence du CD23 sur des cellules épithéliales thymiques de la région souscapsulaire, des cellules stromales de la moelle ct des kératinocytes de la peau a été démontréc réccmment (Mossalayi, Debré et al.). 


\section{Cellules tumorales}

- Néoplasies B. Le CD23 est quasiment toujours présent à haute densité sur les cellules tumorales B des LLC-B. A la différence des lymphocytes $\mathrm{B}$ quiescents, on détecte dans ces cellules les ARNm des isoformes $a$ et $b$; cela pose le problème du facteur responsable de l'induction de la forme b, car l'IL-4 n'est généralement pas active sur ces cellules. Le CD23 est aussi fréquemment exprimé sur des cellules tumorales de lymphomes du médiastin à cellules claires. et de lymphomes folliculaires centrocytiques et, faiblement, dans environ $50 \%$ des leucémies à tricholeucocytes. Il est généralement absent des lignées dérivées de myélome humain.

14. Bruynzeel-Koomen C, Van der Don EM, Bruynzeel PLB, Capron M, De Gas GC, Mudde GC. Associated expression of $\mathrm{T} 6$ antigens and Fc-receptors for $\mathrm{IgE}$ on epidermal Langerhans cells from patients with atopic dermatitis. Clin Exp Immunol $1988 ; 74$ : 137-44.

15. Cordier M, Calender A, Billaud M, $e$ al. Stable transfection of Epstein-Barr virus (EBV) nuclear antigen 2 in lymphoma cells containing the EBV P3HR1 genome induces expression of B-cell activation molecules CD21 and CD23. J Virol 1990; 64 : 1002-13.

16. Wang F, Gregory C, Sample C, et al. Epstein-Barr virus latent membrane protein (LMP1) and nuclear protein-2 and protein-3C are effectors of phenotypic changes in lymphocytes B. EBNA-2 and LMP1 cooperatively induced CD23. J Virol 1990 ; 64 : 2309-18

17. Billaud M, Busson $\mathrm{P}$, Huang $\mathrm{D}$, et al. Epstein-Barr virus (EBV)-containing nasopharyngeal carcinoma cells express the Bcell activation antigen BLAST-2/CD23 and low levels of the EBV receptor CR2.J Virol $1989 ; 63: 4121-8$.

18. Sharma S, Morgan J, Shrayer D, Maizel A, Kumar A, Jackson J. Human BCGF-12 kD and its role in B cell neoplasia. Proc Ist International Congress on Cytokines. Basic principles and clinical application. Florence, 1990.

19. Gordon J, Rowe M, Walker L, Guy G. Ligation of the CD23, p45 (BLAST-2, EBVCS) antigen triggers the cell-cycle progression of activated B lymphocytes. Eur J Immunol $1986 ; 16$ : 1075-80.

20. Swendeman SL, Thorley-Lawson D Soluble CD23/BLAST-2 (sCD23/BLAST-2) and its role in B cell proliferation. Curr Top Microbiol Immunol 1988; 141: 157-64.

21. Uchibayashi $\mathrm{N}$, Kikutani $\mathrm{H}$, Barsumian EL, et al. Recombinant soluble Fce receptor II (FceRII/CD23) has IgE binding activity but no B cell growth promoting
- Cellules transformées par l'EBV. In vitro, l'infection par EBV peut, soit immortaliser les lymphocytes B (lignées lymphoblastoïdes B CD23 + / EBNA ${ }^{+}$), soit les activer pour la sécrétion d'immunoglobulines (B CD23-/EBNA - ). Des expéricnces de transfection dans des cellules $\mathrm{EBV}$ - ont révélé que deux gènes de l'EBV, agissant en coopération, sont nécessaires pour l'immortalisation et l'acquisition du CD23: le LMP1 (latent infection membrane protein 1) et 1'EBNA-2 (Epstein-Barr nuclear antigen-2). Le LMP1 induit le CD23b et des molécules d'adhérence LFA-1, LFA-3, (leukocyte function associated), ICAM-1, (intercellular adhesion molecule), alors que l'EBNA-2 induit préférentiellement l'isoforme CD23a, ainsi que CD21, un autre antigène d'activation B $[15,16]$. Dans les cellules de lymphome de Burkitt, dont l'étiologie est associée au virus EBV, on ne décèle que peu ou pas la présence du CD23, alors que celle-ci est détectée dans les cellules épithéliales tumorales dérivées de carcinome naso-pharyngé, cellules hébergeant l'EBV ; dans ce cas toutefois, la présence du CD23 n'est pas liée à l'expression de l'EBNA-2 [17].

- Autres tumeurs. Le CD23 est présent dans des lignées monoblastiques et de leucémies à éosinophiles, où son expression membranaire et la production de la forme soluble sont augmentées par l'IL-4, le PAF (platelet activating factor) et l'IFN- $\gamma$ et inhibés par le TGF- $\beta$ (transforming growth factor $\beta$ ).

\section{Régulation de l'expression du CD23 et de sa forme soluble par des cytokines et des agents pharmacologiques}

\section{Expression membranaire}

- Lymphocytes B. L'IL-4 est la lymphokine essentielle qui règle l'expression du CD23 dans différents types cellulaires. Elle augmente très nettement le CD23 sur les cellules B humaines normales, en même temps que l'IgM membranaire (sIgM) et avant l'entrée en cycle des cellules. Cet effet touche des cellules $\mathrm{B}$ déjà faiblement CD23 + et ne résulte pas d'un recrutement de cellules CD23 - . L'IL-4 agit au niveau transcriptionnel; elle induit l'isoforme FceRIIb (exprimée spontanément sur les lymphocytes $B$ de patients atopiques) et augmente légèrement l'isoforme FceRIIa [5]. Cet accroissement est bloqué par les interférons IFN- $\alpha$ et IFN- $\gamma$. Le LMW-BCGF (low molecular weight $B$ cell growth factor), facteur de progression des lymphocytes $B$, exerce une régulation différente de celle de l'IL-4 en induisant une augmentation de la forme FceR RIb avec diminution transitoire de la forme FceRIIa [18].

L'IL-2, en présence d'anti-IgM, accroît l'expression membranaire du CD23 vraisemblablement en le stabilisant, car on ne détecte pas d'augmentation concomitante de sCD23.

Divers agents mitogènes et des antiIgM accroissent le CD23 sans augmentation simultanée des sIgM ; leurs effets sont amplifiés par des activateurs de la protéine kinase $\mathrm{C}$ comme certains esters de phorbol (TPA) et par le leucotriène $\mathrm{B}_{4}$ $\left(\mathrm{LTB}_{4}\right)$, un métabolite de l'acide arachidonique. Les toxines de Pertussis et de choléra (sous-unité B) sont mitogéniques pour les lymphocytes $\mathrm{B}$; elles augmentent l'expression du CD23 et la libération de sa forme soluble. Cela pourrait expliquer le rôle de la toxine de Pertussis comme adjuvant de la réponse $\operatorname{IgE}$ (voir fonctions du CD23).

Les lymphocytes $\mathrm{T}$ auxiliaires ( $\mathrm{Th}$ CD4 $\left.{ }^{+}\right)$, activés via leur récepteur 
pour l'antigène, induisent le CD23 dans des cellules $\mathrm{B}$ co-cultivées ; cela nécessite une interaction cognitive des deux types cellulaires et pourrait être amplifié par l'IL-4, ou une autre lymphokine encore indéterminée, produite par les Th. L'interaction entre des clones de cellules $\mathrm{Th}$ humaines alloréactives et cellules B allogéniques correspondantes stimule aussi, chez ces dernières, une expression importante du CD23.

- Tumeurs B. Dans des lignées dérivées de lymphome de Burkitt, l'IL-4 induit le CD23 et augmente de façon coordonnée l'expression d'antigènes du complexe majeur d'histocompatibilité (CMH) de classe II (HL-A $D Q)$; ces effets, qui requièrent une néosynthèse d'ARNm et de protéine, sont inhibés par l'IFN- $\gamma$ au niveau transcriptionnel et par les prostaglandines $\left(\mathrm{PGE}_{2}\right)$ via l'AMP cyclique induit. Dans les LLC-B, l'IL-4 augmente l'expression membranaire et la libération de la forme soluble du CD23, alors que la stimulation via les Ig de surface est inefficace.

- Lymphocytes T. La stimulation par l'allergène induit l'ARNm et la protéine CD23 dans les lymphoblastes T de patients atopiques. Il en est de même avec certains antigènes parasitaires.

- Monocytes. Dans les monocytes humains, l'IL-4 accroît aussi le CD23 et cet effet n'est pas inhibé par l'IFN- $\gamma$. Celui-ci augmente même le CD23 dans des lignées myélomonocytaires. L'IFN- $\alpha$ et un des métabolites qu'il induit, le (2'-5') oligoadénylate, stimulent également l'expression de CD23 dans des lignées monoblastiques. Des agonistes des récepteurs $\beta 2$-adrénergiques induisent eux aussi un accroissement du CD23. Le médiateur lipidique PAF (platelet activating factor) provoque cet effet et ceci n'est pas lié à une induction d'IL-4; le PAF étant libéré des mastocytes sensibilisés au contact de l'antigène ou des macrophages via la stimulation de récepteurs pour l'IgE, il existe donc des échanges d'information entre immunité spécifique et non spécifique via le système IgE. Notons d'ailleurs qu'augmentation d'expression et relâchement de la forme soluble sont exacerbés dans les lymphocytes de patients atopiques.

- Cellules de Langerhans. Le CD23 est induit sur les cellules de Langerhans de la peau d'individus normaux par l'IL-4 et l'IFN- $\gamma$ qui agissent en synergie.

- Cellules épithéliales. Là aussi, IFN- $\gamma$ et IL-4 accroissent l'expression de CD23 sur une sous-population épithéliale thymique de la corticale superficielle (Debré, communication personnelle). De plus, l'IL-4 est un puissant inducteur de CD23 sur les cellules épithéliales de carcinome nasopharyngé $\mathrm{EBV}+{ }^{+}$, et son action est encore amplifiée par l'anticorps anti-CD40 (voir plus loin) qui accroît aussi le relâchement de la forme soluble sCD23, ainsi que celle de l'IL-1, molécules qui pourraient jouer un rôle dans l'évolution de cette tumeur.

Modulation de la production de CD23 soluble par des cytokines

In vitro, le relâchement spontané de la forme soluble est souvent, mais pas toujours, corrélé à la diminution d'expression membranaire du CD23. L'IL-4 accroît ainsi la sécrétion de la forme soluble par les monocytes normaux, la lignée monoblastique U937 et les lymphocytes B normaux. Pour ces derniers, le sCD23 produit est identique à celui relâché spontanément par des lignées lymphoblastoïdes B. L'IFN- $\gamma$ a des effets antagonistes suivant les types cellulaires; dans les cellules B, il inhibe la stimulation par l'IL-4 de la forme membranaire du CD23 et la sécrétion de sa forme soluble, mais seul, il induit le relâchement de sCD23 dans les monocytes. Quant à l'interféron $\alpha$, il inhibe la formation de CD23 soluble induite par IL-4 ou IFN- $\gamma$.

\section{Fonctions des molécules CD23 membranaires et solubles}

Le CD23 membranaire récepteur d'un facteur de croissance des lymphocytes B ?

Certains anticorps monoclonaux antiCD23 induisent la synthèse d'ADN dans des cellules B costimulées par un ester de phorbol et miment l'effet du LMW-BCGF. La proposition que le CD23 serait le récepteur de ce fac- teur n'a pas été confirmée, mais il est toutefois très vraisemblable que le CD23 soit le récepteur d'une lymphokine, non encore identifiée, impliquée dans le contrôle de la prolifération B [19]. Par ailleurs, des anticorps dirigés contre des antigènes de classe II du CMH bloquent la prolifération spontanée de lignées lymphoblastoïdes B immortalisées par l'EBV et cette inhibition s'accompagne d'une diminution du CD23. Ce fait, joint à l'existence bien établie d'un facteur de croissance (BCGF autocrine) opérant dans ces cellules, conforte l'hypothèse que le CD23 et/ou son fragment soluble sCD23 sont importants dans le contrôle de la prolifération de ces cellules. Deux hypothèses sont envisageables: le CD23 sert de récepteur à un BCGF autocrine ou le sCD23 est lui-même le facteur autocrine.

\section{Le sCD23 facteur de progression} des lymphocytes $B$ ?

Swendeman et Thorley-Lawson ont montré qu'un complexe soluble des formes naturelles de clivage $25 \mathrm{kDa}$ et $12 \mathrm{kDa}$ du CD23 induit la prolifération de cellules $B$ normales activées et de lignées transformées par l'EBV [20]. D'autres auteurs ont décrit une activité mitogénique associée à la forme membranaire de $45 \mathrm{kDa}$ ou au fragment soluble de $37 \mathrm{kDa}$ ou à celui de $12 \mathrm{kDa}$. En revanche, d'autres groupes n'ont pu déceler d'activité mitogénique du fragment sCD23 recombinant de $25 \mathrm{kDa}$, ni des autres fragments recombinants sCD23 liant l'IgE [21, 22]. Ces divergences pourraient s'expliquer par l'instabilité de certains fragments solubles et par la nécessité que plusieurs de ces fragments coopèrent entre eux.

Récemment, il a été montré que l'on pouvait établir des lignées de lymphocytes $\mathrm{B}$ normaux en culture à long terme au moyen de la combinaison IL-4 et d'anticorps dirigés contre le CD40 [23, 24]. Cette molécule possède des analogies avec le récepteur du NGF (nerve growth factor) et du TNF (tumor necrosis factor); son ligand physiologique est activement recherché et pourrait être un constituant membranaire des lymphocytes $T$ ou des cellules folliculaires dendritiques [23]. Il a été suggéré que 


\section{RÉFÉRENCES}

22. Luo $\mathrm{H}$, Hof stetter $\mathrm{H}$, Banchereau J, Delespesse G. Cross-linking of CD23 antigen by its natural ligand (IgE) or by antiCD23 antibody prevents B lymphocyte proliferation and differentiation. I Immunol 1991 (sous presse).

23. Banchereau J, De Paoli P, Vallé A, Garcia E, Rousset F. Long-term human B cell lines dependent on interleukin 4 and antibody to CD40. Science 1991; 251 : 70-2.

24. Banchereau J. Interleukine 4. médecine/sciences $1990 ; 10$ : 946-53.

25. Liu YJ, Joshua DE, Williams GT, Smith CA, Gordon J, MacLennan ICM. Mechanism of antigen-driven selection in germinal centres. Nature $1989 ; 342$ : 929-31.

26. Sarfati M. Delespesse G. Possible role of human lymphocyte receptor for $\mathrm{IgE}$ (CD23) or its soluble fragments in the in vitro synthesis of human IgE. I Immunol $1988 ; 141: 2195-9$.

27. Capron A, Dessaint JP, Capron M, Joseph M, Ameisen JC, Tonnel AB. From parasites to allergy: a second receptor for IgE. Immunol 7iday 1986; 7 : 15-8

28. Ciapron A, Dessaint JP. Présent et futur de l'allergie. médecine/sciences $1990 ; 10$ : 958-64.

29. Kehry MR, Yamishita LC. Fce receptor II (CD23) function on mouse B cells : role in $\operatorname{IgE}$ dependent antigen focusing. Proc Natl Acad Sci USA 1989 ; 86 : 7566-70.

30. Kolb JP, Renard D, Dugas B, et al. Monoclonal anti-CD23 antibodies induce a rise in $\left[\mathrm{Ca}^{2}+\right]_{\mathrm{i}}$ and polyphosphoinositide hydrolysis in human activated B cells : involvement of a $\mathrm{Gp}$ protein. I Immunol $1990 ; 145: 429-34$

31. Mossalayi D, Lecron JC, Dalloul AH, et al. Soluble CD23 (FcREII) and IL-1 synergistically induce early human thymocyte maturation. J Exp Med 1990 ; 171 : 959-64.

32. Flores-Romo L, Cairns JA, Millsum MJ, Gordon J. Soluble fragments of the low-affinity $\operatorname{IgE}$ receptor (CD23) inhibit the spontaneous migration of U937 monocytic cells : neutralization of MIFactivity by a CD23 antibody. Immunology $1989 ; 67: 547-9$.

33. Flores-Romo L, Johnson GD, Ghaderi AA, Stanworth DR, Veronesi A, Gordon J. Functional implication for the topographical relationship between $\mathrm{MHC}$ class II and the low-affinity IgE receptor ; occupancy of CD23 prevents B lymphocytes from stimulating allogeneic mixed lymphocyte responses. Eur J Immunol 1990 ; le sCD23, induit dans ces conditions, pourrait jouer un rôle de facteur autocrine pour ces lignées B à long terme [24].

Par ailleurs, la stimulation de lymphocytes B via le CD40 (ou par les Ig de membrane) prévient aussi le phénomène d'apoptose, mort cellulaire programmée, qui affecte les lymphocytes B centroblastiques des centres germinatifs lors du processus d'hypermutation somatique conduisant à la maturation de l'affinité des anticorps [25]. Le relâchement de sCD23 par les cellules folliculaires dendritiques pourrait également contribuer, en synergie avec l'IL- $1 \alpha$, à prévenir ce phénomène d'apoptose. Notons aussi que des cellules $T$ d'amygdales activées par des mitogènes, l'IL-4 ou un ester de phorbol, voient leur prolifération avec ces agents augmenter en présence d'anticorps anti-CD23 ou de CD23 soluble ; le CD23 exerce donc une action sur la régulation de leur prolifération [12].

\section{sCD23 et pathologie}

Les sérums (et les urines) d'individus normaux contiennent une faible concentration d'IgE-BF. Cette concentration est augmentée chez les patients atteints de syndrome hyper-IgE ou de dermatite atopique, où on les trouve souvent sous forme de complexes avec l'IgE.

Le sérum des patients atteints de LLC-B contient des quantités très élevées de sCD23, corrélées à la sévérité de la maladie, suivant la classification histologique de $\mathrm{Rai}$ et au compte lymphocytaire. Les patients tricholeucémiques ont aussi des taux sériques élevés de sCD23, qui se normalisent rapidement lors du traitement par interféron; l'effet thérapeutique de l'IFN- $\alpha$ pourrait être relié à cet effet, si l'on suppose que le sCD23 exerce effectivement une action de prolifération sur les tricholeucocytes. Même si ce rôle de facteur autocrine du sCD23 pour des tumeurs B n'est pas clairement établi, la mesure de ce paramètre pourrait se révéler d'un grand intérêt dans la surveillance de ces affections.

\section{Rôle dans la synthèse des IgE} Plusieurs résultats suggèrent fortement que le CD23 et/ou ses frag- ments solubles jouent un rôle important dans la biosynthèse de l'IgE. (1) Le surnageant de lignées $B$ humaines CD23 + contient une activité stimulant la production d'IgE par des lymphocytes B isolés du sang de patients atopiques; cette activité est absorbée à la fois par des colonnes d'IgE et par des colonnes d'anticorps anti-CD23 d'où on peut la récupérer par élution et montrer que cet $\mathrm{IgE}-\mathrm{BF}$ résulte effectivement du clivage du CD23.

(2) Un des effets majeurs de l'IL-4, sa capacité à induire in vitro la production d'IgE par des lymphocytes humains normaux, est inhibée par des anticorps anti-CD23 [26]. La biosynthèse d'IgE par des lymphocytes B, spontanée chez les patients atopiques ou induite par l'IL-4 chez les donneurs normaux, est supprimée par des anti-CD23 reconnaissant le site de liaison à $\mathrm{l}$ 'IgE.

(3) L'IL-2 augmente en parallèle la synthèse d'IgE et d'IgE-BF/sCD23 dans des lymphocytes stimulés par un mitogène bactérien, le SAC (Staphylococcus aureus Cowan I).

(4) Chez la majorité des patients atteints de diverses formes de leucémies aiguës et ayant subi une autogreffe de moelle osseuse, on observe un pic transitoire d' $I g E$ sérique trois à quatre semaines après la greffe, celui-ci étant précédé de quelques jours par une augmentation importante du taux de sCD23 ; la provenance de celui-ci n'a pu être identifiée, mais cette observation renforce la suggestion d'un rôle effectif in vivo du sCD23 dans la biosynthèse de l'IgE. (5) Étant donné les liens bien connus entre syndromes hyper-IgE et éosinophilie, il est possible que du sCD23 dérivé d'éosinophiles ait un rôle régulateur sur la production d'IgE.

Toutefois, il existe des rapports contradictoires quant à la capacité du sCD23 purifié à moduler la synthèse d'IgE. Chez la souris, le sCD23 est sans effet sur la synthèse d'IgE; il faut néanmoins rappeler que les fragments murins sCD23 ne lient pas (ou peu) l'IgE. Chez l'homme, un travail effectué avec le fragment recombinant de $25 \mathrm{kDa}$ conclut à son absence d'effet sur la synthèse d'IgE. En revanche, d'autres études ont montré un effet stimulant très net de 
sCD23 purifié, naturel ou obtenu par génie génétique, le fragment actif étant la forme de $37 \mathrm{kDa}$. Un mécanisme possible serait que le sCD23 agisse sur des cellules $\mathrm{B}^{\mathrm{sIgE}}{ }^{+}$ayant déjà subi la commutation isotypique et qu'il accroisse leur production d'IgE ; rappelons cependant que les précurseurs des cellules $B$ sécrétrices d'IgE proviennent surtout de lymphocytes B $\operatorname{sig} \mathrm{M}^{+} \operatorname{sIgE} \mathrm{E}^{-}$.

Le sCD23 pourrait aussi stimuler la sécrétion d'IL-4 par les cellules T auxiliaires (Th) et potentialiser la réponse $\mathrm{IgE}$, surtout quand l'IL-4 est en concentration sub-optimale. Signalons que l'IL-5 et le leucotriène $\mathrm{B}_{4}$ $\left(\mathrm{LTB}_{4}\right)$ potentialisent l'action de l'IL-4 sur la synthèse d'IgE et augmentent en parallèle le relâchement de sCD23, ces deux effets étant bloqués par l'IFN- $\gamma$ et par des anticorps anti-CD23. L'importance du CD23 dans la réponse IgE est encore renforcée par l'observation que la liaison au CD23 de complexes IgE-antiIgE ou d'anticorps anti-CD23 provoque, dans les lymphocytes B de patients atopiques (et dans un myélome sécréteur d'IgE), une inhibition spécifique d'isotype de la biosynthèse spontanée d'IgE par ces cellules. La fixation de complexes immuns à $\mathrm{IgE}$ sur le CD23 pourrait favoriser son internalisation et sa dégradation au détriment de la production de sCD23 soluble, ce qui conduirait à une inhibition de la synthèse d'IgE. Comme il existe des anticorps anti-IgE dans le sérum de patients présentant des allergies cutanées ou respiratoires, un tel mécanisme pourrait donc être opérationnel in vivo. Notons d'ailleurs que le niveau, élevé, de CD23 sur les cellules B de patients allergiques retourne à la normale au cours de la désensibilisation.

Rôle dans la défense antiparasitaire Diverses cellules de l'inflammation macrophages, éosinophiles et plaquettes - sont des cellules effectrices de l'ADCC (cytotoxicité cellulaire dépendante d'anticorps) envers les parasites. Ce type de lyse fait intervenir des anticorps anti-parasites du type de ceux retrouvés dans les manifestations d'hypersensibilité immédiate et allergique, essentiellement l'IgE [27]. Cette ADCC s'effectue par l'entremise du CD23, souvent en association fonctionnelle avec la molécule CD11b (récepteur du chaînon $\mathrm{iC}_{3} \mathrm{~b}$ du complément). La reconnaissance, via des $\operatorname{IgE}$ spécifiques des antigènes du parasite, déclenche chez les cellules effectrices le relâchement de divers médiateurs, tels prostaglandines, PAF, métabolites de $\mathrm{O}_{2}$, etc.

Rôle dans les réactions allergiques La manifestation allergique résulte de divers processus impliquant la synthèse d'IgE [28]. L'interaction de l'antigène (allergène) avec l'IgE fixée sur le FceRI des mastocytes et des basophiles induit des signaux membranaires conduisant à la dégranulation de ces cellules. Celles-ci libèrent une série de médiateurs, amines vasoactives et composés lipidiques (leucotriènes, PAF) responsables des réactions immédiates tel le choc anaphylactique, mais aussi des cytokines comme l'IL-4. L'IL-4 ainsi produite est capable d'induire le CD23 dans des cellules environnantes. Cet effet est fortement amplifié par le PAF, le LTB4, et la production d'IgE, déclenchée par l'IL-4, est augmentée par ces médiateurs lipidiques. Les macrophages alvéolaires, lors d'allergie des voies aériennes, sont induits à exprimer le CD23 et peuvent donc ultérieurement interagir avec des complexes immuns à $\mathrm{IgE}$, libérant à leur tour divers médiateurs de l'inflammation, processus conduisant à la survenue des manifestations retardées de la réaction allergique.

\section{Présentation de l'antigène via le CD23}

Les lymphocytes B, par l'intermédiaire du CD23 et d'IgE spécifiques, sont capables de présenter l'antigène à des cellules $\mathrm{T}$ auxiliaires et de provoquer leur activation, avec une efficacité comparable à celle des immunoglobulines de membrane [29]. Il est vraisemblable que cette fonction cruciale est également opérationnelle au niveau d'autres cellules CD23 + présentatrices de l'antigène, comme les cellules folliculaires dendritiques et les cellules de Langerhans.

Rôle dans l'adhérence cellulaire La transfection de cellules CD23 par le gène du CD23 induit la formation d'agrégats cellulaires, suggé- rant un rôle du CD23 dans les phénomènes d'adhérence, impliquant la séquence consensus RGD. Signalons aussi que, dans les plaquettes, le CD23 est associé à la molécule GPIIb-IIIa, adhésine récepteur du fibrinogène. De plus, le CD23 présente des homologies via le domaine des lectines, avec des molécules de type homing receptor (récepteur de domiciliation des lymphocytes) (MEL-14, LAM-1, ELAM-1).

Transduction et signalisation intracellulaire via le CD23

Des anticorps anti-CD23 induisent l'hydrolyse des polyphospho-inositides et une mobilisation du calcium intracellulaire dans les lymphocytes B activés pour l'expression de CD23 ; ce processus implique une protéine liant le GTP (G-protéine), insensible à la toxine de Pertussis [30]. La fixation de l'IgE induit aussi ces événements précoces, pourvu qu'on provoque son pontage avec un anti-IgE. Comme le CD23 n'a pas la structure classique à sept domaines transmembranaires des récepteurs couplés aux protéines $G$, cela suggère qu'il pourrait être associé à d'autres protéines de surface, comme cela a été montré pour les Ig membranaires. En fonction des types cellulaires exprimant le CD23, ces associations pourraient être différentes et conduire à des modes de transduction variés. Notons d'ailleurs que l'association du CD23 avec une protéine tyrosine kinase a été rapportée par Yodoï et nos résultats suggèrent que la signalisation via CD23 dans les monocytes est différente de celle observée dans les lymphocytes B. Mentionnons aussi que les isoformes $a$ et $b$, qui n'ont pas le même domaine cytoplasmique, pourraient transduire des signaux différents.

Effet du sCD23 sur la différenciation hématopoïétique

En synergie avec l'IL-1, le sCD23 de $25 \mathrm{kDa}$ induit la maturation de prothymocytes humains en thymocytes différenciés, capables de répondre à des stimuli variés [31]. In vivo, le sCD23 libéré par des cellules épithéliales thymiques jouerait donc un rôle important dans l'acquisition de la compétence immunitaire des lymphocytes T. En synergie avec 
l'IL-1, un effet prolifératif et différenciant du sCD23 sur des précurseurs myéloïdes, notamment vers la voie des basophiles, a été également observé par le même groupe.

\section{Rôle du sCD23 dans la migration cellulaire}

Des préparations de sCD23 sont mitogéniques et exercent une activité de type MIF (migration inhibitory factor) sur les cellules tumorales monoblastiques U937. Cela suggère un rôle possible du CD23 sur la mobilité des monocytes/macrophages [32].

\section{Conclusion}

Le CD23 est une molécule qui exerce, via sa forme membranaire ou ses fragments solubles, une variété de fonctions sur différentes cellules du système immunitaire à divers stades de leur maturation. En particulier, des fragments de clivage libérés par des cellules épithéliales thymiques et de stroma médullaire interviennent dans la différenciation précoce de précurseurs myéloïdes et des lymphocytes T. Plus tardivement, des fragments solubles relâchés par des cellules folliculaires sont impliqués dans la maturation terminale des lymphocytes B.

S'il est trop tôt pour proposer un schéma intégrant toutes les propriétés attribuées au CD23, on peut cependant émettre quelques hypothèses pour ses fonctions liées à la présentation de l'antigène et à la régulation de la réponse IgE. Dans un premier temps, l'IgE circulante (produite lors d'une réponse primaire) se lie au CD23 présent sur les lymphocytes $\mathrm{B}$; en ralentissant son catabolisme, elle augmente les chances de rencontre avec l'antigène spécifique $(\mathrm{Ag})$. La fixation de celui-ci provoque la formation d'un complexe $\mathrm{IgE} / \mathrm{Ag}$, dont la vitesse de dissociation du CD23 est plus faible que celle de l'IgE libre. Cela favorise les chances de l'ensemble ternaire (CD23/IgE/Ag) d'être internalisé, par analogie avec ce que l'on sait des lectines animales homologues du CD23 (récepteur de l'asialoglycoprotéine), qui internalisent leur ligand une fois fixé et l'amènent dans des compartiments de dégradation. Le complexe compte tenu de la labilité du fragment Fc de l'IgE au $\mathrm{pH}$ acide existant dans ces vacuoles. L'antigène est dégradé et des peptides antigéniques sont captés par des molécules du $\mathrm{CMH}$ de classe II qui transitent dans ces vacuoles en provenance du Golgi. Il y aurait ensuite co-expression à la membrane de ces molécules chargées en peptides antigéniques avec le CD23 réexprimé : une co-localisation des deux molécules à un même pôle de la cellule a d'ailleurs été observée récemment [33]. Ensuite, une cellule $\mathrm{Th}$ auxiliaire (helper) CD4 + reconnaît, via son complexe récepteur de l'antigène ( $\mathrm{T} c \mathrm{R})$, l'antigène ainsi présenté par le CMH II. Le CD23 présent sur la cellule B pourrait favoriser cette reconnaissance en jouant un rôle de molécule d'adhérence auxiliaire, car son domaine d'homologie avec les lectines animales est similaire à celui de plusieurs homing receptors, et éventuellement fournir lui aussi un signal d'activation à la cellule $T$. Les lymphocytes $T h$ une fois activés deviennent capables de libérer dans leur environnement de l'IL-4, qui augmenterait l'expression du CD23 sur d'autres lymphocytes $\mathrm{B}$, induisant ainsi une boucle d'auto-amplification pour la présentation de l'antigène aux cellules $T$. La fréquence des cellules CD23 + est très supérieure à celle des lymphocytes $B$ spécifiques de l'antigène, d'où une amplification de la stimulation des $\mathrm{Th}$. Le problème se pose alors de savoir si ces lymphocytes B sont eux-mêmes activés par leur interaction avec les $\mathrm{Th}$, car cela conduirait à une production polyclonale d'anticorps non spécifiques de l'antigène. Un mécanisme de rétrocontrôle est toutefois suggéré sur la base de résultats très récents. Ceux-ci montrent que le cross-linking du CD23, à la surface des précurseurs B, par des IgE complexées à l'antigène, inhibe les effets de l'IL-4 sur la prolifération et la différenciation en cellules sécrétrices d'IgM et d'IgE, alors que la sécrétion d'IgG n'est pas altérée ([25] et Delespesse, communication personnelle). Cela se comprend, puisque les précurseurs des cellules synthétisant les $\operatorname{IgM}$ et les $\operatorname{IgE}$ sont essentiellement sIgM + sIgE-/CD23+, alors que les cellules ayant déjà subi la commutation isotypique pour la synthèse d'IgG sont sIgG + /CD23 - . Ce processus conduirait donc à une suppression de la synthèse d' $\operatorname{IgE}$ et à une promotion de la synthèse des autres isotypes par les cellules $\mathrm{Th}$ ainsi amplifiées.

Par ailleurs, il a été montré expérimentalement que de l'IgE libre, un de ses fragments liant le CD23, ou un anticorps anti-CD23 dirigé contre le site de liaison de $\operatorname{l'} \operatorname{IgE}$, bloquent la présentation par les lymphocytes B d'alloantigènes aux lymphocytes $T$ [33]. L'occupation du site IgE du CD23 par ces agents pourrait donc interférer avec le processus décrit précédemment, soit à son début, en entrant en compétition avec la fixation de l'IgE spécifique de l'antigène, soit par empêchement stérique au moment de la présentation des peptides antigéniques aux cellules $\mathrm{T}$. Notons que ce dernier stade est également bloqué par des anticorps anti-classe II.

Ces données suggèrent que physiologiquement, une fois l'excès d'antigène éliminé, l'IgE libre, qui n'est pas ou peu internalisée après fixation sur le CD23, induirait une inhibition rétroactive par ce mécanisme. Suivant le degré de complexation à l'antigène, l'IgE pourrait donc moduler dans les deux sens la capacité du CD23 à fonctionner comme un relais dans la présentation de peptides antigéniques par les molécules du $\mathrm{CMH}$ de classe II.

Contrôle de la prolifération des lymphocytes $B$ et régulation de la synthèse de l'IgE représentent vraisemblablement deux facettes d'un même processus dans lequel, en liaison avec l'IL-4, est impliqué le CD23. Étant donné ses fonctions dans la biosynthèse et les fonctions effectrices de l'IgE, on comprend mieux l'intérêt énorme que cette molécule suscite dans les domaines de l'allergologie et de la parasitologie. Bien que, pour l'instant, difficile à relier à ses autres propriétés, le rôle que joue le CD23 dans la différenciation de précurseurs hématopoïétiques est aussi d'un intérêt crucial. Les questions que soulèvent les multiples fonctions du CD23 font l'objet de recherches intensives. De façon non exhaustive, citons la recherche d'un éventuel second ligand (facteur de croissance des lymphocytes B ?), 
l'étude des mécanismes liés à l'immortalisation par l'EBV, les seconds messagers induits, la régulation de l'activité autocatalytique, le rôle dans les fonctions d'adhérence et de mobilité cellulaire, l'existence et la caractérisation de récepteurs spécifiques pour les différentes formes de sCD23. Nul doute que l'avenir proche nous réserve encore des surprises sur cette molécule pléïotrope

\section{Summary}

CD23, a molecule with multiple functions

The low affinity IgE receptor CD23 molecule has gained considerable popularity during the recent years. The membrane form or the soluble cleavage fragments of this molecule appear to be involved in several immunological processes, most of ten in relation with interleukin 4. These events include control of B cell proliferation, capacity to present antigen to $T$ cells through specific IgE antibodies, selective killing of some parasites and regulation of IgE biosynthesis. The latter point is of peculiar interest since it offers some clues about possible means to control adverse allergic reactions. In addition, CD23 contributes to the differenciation of pre-T and other hematopoietic cell precursors and could also be implicated in cellular adhesion. Moreover, the cleavage of the molecule, which seems to be autocatalytic, raises intriguing questions about the mechanisms which control this enzymatic process.

\section{Remerciements}

Les auteurs remercient l'Inserm, l'ARC et l'Institut Henri-Beaufour pour leur soutien.

TIRÉS A PART

J.-P. Kolb.

$\mathrm{m} / \mathrm{s} n^{\circ}$ 5, vol. 7, mai 91 